\title{
Study of non-linear mechanical behavior of oil palm mesocarp fibers
}

\begin{abstract}
This work investigates the non-linear mechanical behavior of oil palm mesocarp fibers (OPMF) using tensile tests, microstructure observation, and finite element models. The micrograph images showed the fiber's surface with partly embedded silica bodies, while the cross section contained cell wall structures. Viscoelastic behavior was observed when the fibers were relaxed over time after being stretched, whereas the stress--strain curves from the cyclic tests indicated permanent set (plastic strain) due to the fibers' deformation. Finite element models were developed comprising single particles (2D and 3D) and 2D multiparticle geometries representing silica bodies embedded in a matrix representing the fiber. The modeling results suggested that silica bodies do not contribute much to the integrity of OPMF, highlighting the need to have a more complex model that considers cellular structures of the fibers and a constitutive relationship of cellulose, hemicelluloses, and lignin.
\end{abstract}

Keyword: Cohesive zone modelling; Finite element method; Oil palm mesocarp fibers; Viscoelastic 
